\title{
Verso il modello integrato della gestione dell'accesso vascolare per emodialisi
}

Pur tra le difficoltà quotidiane che tutti noi dobbiamo affrontare, la qualità dell'accesso vascolare per dialisi creato e gestito negli ospedali italiani resta tra le migliori del mondo. Tuttavia, gli importanti (e veloci) cambiamenti della sanità impongono un continuo adeguamento che inevitabilmente cambia abitudini e assetti organizzativi consolidati. Si potrebbe dire che è giunto ormai il momento in cui il nefrologo superi da un lato 'l'autarchia' che lo ha contraddistinto per molto tempo e dall'altro la tendenza alla delega completa nella gestione degli accessi vascolari per emodialisi e abbracci un modello collaborativo con altri specialisti per ottenere un'organizzazione diversa e più efficiente. Non si tratta di una riduzione del ruolo del nefrologo. Al contrario, in un modello di effettiva collaborazione tra diversi attori, al nefrologo deve restare il ruolo del 'regista'.

L'editoriale di Luciano Carbonari, Franco Galli e Luigi Tazza, esprime molto chiaramente la oramai sopraggiunta necessità di passare da un modello monospecialistico o plurispecialistico non integrato a uno polispecialistico-integrato, ovvero dove gli attori siano «vicini» e soprattutto «capaci di recitare a soggetto seppur coordinati da una sapiente regia». Sia concesso a chi scrive di ribadire, in stretto accordo con gli Autori, che tra gli attori principali oltre al chirurgo vascolare, al radiologo interventista, all'infettivologo e al nefrologo anche il paziente e non certo ultimi gli infermieri di dialisi debbano svolgere la loro parte, a dir poco, importante. Infatti in altri Paesi il ruolo di coordinatore degli accessi vascolari è svolto spesso dal personale infermieristico e anche in Italia si sente la necessità di un progressivo maggiore conivolgimento degli infermieri nella gestione clinico-organizzativa del paziente dializzato.

Se si accetta come fondamentale una condivisione diretta tra gli attori già menzionati, la conseguenza è che la condivisione delle problematiche degli accessi vascolari dovrebbe esser svolta tra queste figure in spazi (compresa la sala dialisi) e tempi ben definiti.

$\mathrm{Ci}$ aspettiamo che le aziende sanitarie facilitino l'integrazione del team-polispecialistico e ne riconoscano l'importanza per ottenere buoni esiti clinici e una migliore qualità dell'assistenza. Un team integrato ben funzionante saprà ottenere effettivi e tangibili risultati ed essere costo-efficace.

Il modello che gli Autori descrivono e auspicano nel loro contributo, per chi scrive dovrebbe essere adottato e diffuso quanto prima possibile nell'interesse di tutti ma soprattutto dei pazienti.

$\mathrm{Ci}$ sentiamo di raccomandare a tutti un'attenta lettura di questo contributo, che suscita inevitabilmente una profonda riflessione che speriamo riesca a smuovere le coscienze e gli interessi di tutti quanti vi sono citati, non ultimi ovviamente gli amministratori della sanità.

Maurizio Gallieni

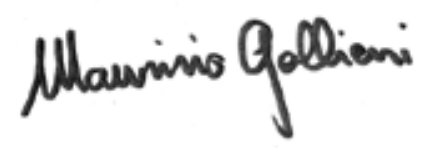

Marco Lombardi

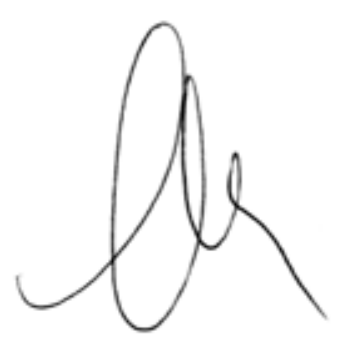

Marcello Napoli

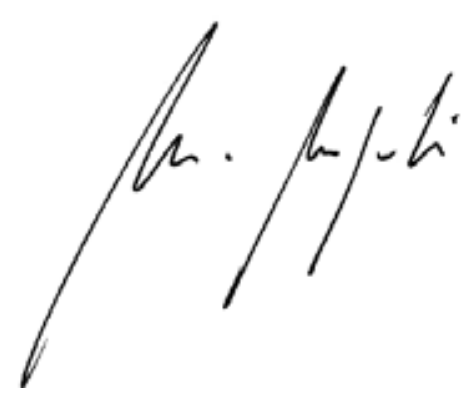

